This document is the accepted manuscript version of the following article:

Marchionni, V., Ferri, D., Kröcher, O., \& Wokaun, A. (2017). Increasing the sensitivity to short-lived species in a modulated excitation experiment. Analytical chemistry, 89 (11), 5801-5809. https://doi.org/10.1021/acs.analchem.6b04939

\title{
Increasing the sensitivity to short-lived species in a modulated excitation experiment
}

\author{
Valentina Marchionni, ${ }^{*, \dagger}, \ddagger$ Davide Ferri, ${ }^{\dagger}$ Oliver Kröcher, ${ }^{\dagger}, \uparrow$ and Alexander \\ Wokaun $*,+\ddagger$ \\ †Paul Scherrer Institut, CH-5232 Villigen PSI, Switzerland \\ $\ddagger$ ETH Zürich, Institute for Chemical and Bioengineering, CH-8093 Zürich, Switzerland \\ 【École polytechnique fédérale de Lausanne (EPFL), Institute of Chemical Science and \\ Engineering, CH-1015 Lausanne, Switzerland \\ E-mail: valentina.marchionni@psi.ch; alexander.wokaun@psi.ch
}

Phone: +41 (0)56 310 2137; +41 (0)56 3102751

\begin{abstract}
The combination of spectroscopic and diffraction methods to study chemical transformations is fundamental for the understanding of reaction mechanisms. The identification of short-lived species, likely active species, is often hindered by the contribution of spectator species not directly involved in the reaction. The present study considers two different approaches to obtain increased sensitivity to transient species for experiments obeying the modulated excitation paradigm and exploiting phase sensitive detection (PSD). First, the variation of the frequency of the external stimulation $(\omega)$ during the experiment is considered. We demonstrate using the Fourier analysis, that the increase of $\omega$, in other words the decrease of the modulation period $T$, enhances the sensitivity to short-lived species. The second alternative is the employment of a single stimulation frequency $(\omega)$ during the measurement and the variation of the demodulation frequency


$(n \omega)$ during data analysis. The absolute intensity of the phase-resolved signals is reduced by increasing $n$. However, species with slow kinetics are more attenuated than species with fast kinetics. Thus, transient species possessing fast kinetics are enhanced relative to other components and can be in principle discerned with improved sensitivity in the phase-resolved data obtained with $n>1$. Experimental results in the field of heterogeneous catalysis are provided that support our findings.

\section{Introduction}

In in situ and operando experimentation, the spectroscopic or diffraction fingerprint of spectator species that are not directly involved in a chemical reaction, including for example solvents or catalyst supports, is frequently stronger than that of active species involved in the catalytic process, thus masking the meaningful information. Therefore, the identification of the active species and the discrimination from the spectator species is difficult. Transient analytical methods can be exploited to facilitate this task. Transients are introduced into a system by perturbing one or more state variables a single time or multiples times. For example, temporal analysis of products (TAP) ${ }^{1,2}$ and steady state isotopic transient kinetic analysis (SSITKA) ${ }^{3}$ experiments exploit pulses and step changes in the reactant gas concentration, respectively, to probe the response of relevant species and to gain mechanistic information on gas-solid catalytic reactions while a spectroscopic experiment is performed. In frequency response techniques, ${ }^{4,5}$ the reacting system is subjected to periodical perturbations. All components of the system that are affected by the change of this parameter change also periodically at the same frequency of the stimulation, or harmonics thereof. A retardation (phase lag $\varphi$ ) between excitation and response may appear, which contains kinetic information. Examination of a catalytic system under periodic operation can provide insight into kinetics and reaction mechanisms above and beyond that achievable with steady state measurements. ${ }^{5-9}$ A mathematical description of isotopic frequency response was derived $^{10}$ to combine the advantages of the frequency response method and of SSITKA. A 
short exciting pulse of light and modulated light at variable frequency are commonly used to recover the lifetimes of a fluorescent sample in pulse and phase-modulation fluorimetry, respectively. ${ }^{11}$

Another strategy for transient experiments consists in the combination of the modulated excitation (ME) approach with phase sensitive detection (PSD). ${ }^{12}$ The periodic perturbation (modulation) of an external parameter is employed to stimulate the system under observation while time-resolved data are collected simultaneously. The modulation approach can be applied if the system responds reversibly to the external periodic excitation. After an initial time, the system reaches a quasi equilibrium state under the applied modulated stimulation. Averaging the data collected over several modulation periods from this point onwards provides already an improvement in the signal-to-noise ratio. ${ }^{13,14}$ The PSD algorithm ${ }^{12}$ is then applied to the set of time-resolved data after data acquisition in order to further enhance the sensitivity of the analytical method. The data are treated using the following equation to obtain a new set of phase-resolved (demodulated) data:

$$
I_{n}\left(E, \phi^{P S D}\right)=\frac{2}{T} \int_{T} I(E, t) \cdot \sin \left(n \omega t+\phi^{P S D}\right) d t
$$

where $T$ is the modulation period, $I(E, t)$ is the intensity of the signal as function of energy $E$ and time $t, \omega$ is the frequency of the external stimulation, $n \omega$ is the demodulation frequency, and $\phi^{P S D}$ is the demodulation phase angle. The integer variable $n=1,2,3 \ldots$ determines the frequency at which the time dependent signals are demodulated, for $n=1$ the demodulation frequency is equal to the stimulation frequency (fundamental frequency), $n=2,3 \ldots$ harmonic frequencies are obtained. The advantage of the phase-resolved dataset is that it contains only the information about the components that respond to the external stimulation. Static signals, e.g. those generated by the catalyst support, the bulk of the material and the spectator species, are cancelled. This leads to the elimination of background signals and to a further improvement of the signal-to-noise ratio. ${ }^{15}$ Moreover, PSD allows the distinction 
of overlapping signals exhibiting different kinetics: ${ }^{15-17}$ the demodulation phase angle can be chosen such that the signal(s) of a particular species vanishes completely while that of another component with different time response remains thus enabling its identification. Thus, the second component can be easily identified and distinguished. In addition, kinetic information can be gained from the analysis of the phase lags. ${ }^{18}$

Urakawa et al. ${ }^{18}$ analysed the advantages of square-wave stimulation over a simple sinusoidal-wave stimulation in modulated excitation spectroscopy. Sinusoidal-wave stimulation is simpler from a mathematical point of view for quantitative analysis; on the other hand, square-wave stimulation is often easier to generate experimentally. According to the Fourier decomposition, a square-wave can be written as the sum of sinusoidal waves of odd frequencies. The demodulated response amplitude obtained for a linear system with sinusoidal-wave stimulation at the $n \omega$ modulation frequency was demonstrated to be equal to the one obtained by square-wave stimulation at the $\omega$ modulation frequency and demodulation at $n \omega$ frequency multiplied by the factor $\pi / 4$ and the demodulation index $n$, where $n$ assumes only integer odd values. The phase lag of the response obtained by square-wave stimulation and demodulation at $n \omega$ frequency is equal to the one obtained by sinusoidalwave stimulation at the $n \omega$ modulation frequency and demodulated at the fundamental frequency. Therefore, a modulated excitation experiment applying square-wave stimulation provides the same information as several experiments applying sinusoidal-wave stimulations at the same frequency $\omega$ of the square-wave stimulation and at higher frequencies $(3 \omega, 5 \omega \ldots)$. There is an upper limit for the demodulation frequency because the amplitudes of higher frequency terms in a square-wave decrease by the factor $n$. The non-linearity of most real systems will make it very difficult to determine the frequency responses exactly. However, when a sufficiently small stimulation is used and a number of modulation cycles is averaged to enhance signal-to-noise ratio and to obtain higher frequency terms of the response with high accuracy, the high frequency demodulation will serve as a good estimate of the responses at high frequencies. 
The validity of the modulation approach and the PSD technique was first demonstrated for in situ attenuated total reflection infrared spectroscopy (ATR-IR), ${ }^{15,19,20}$ polarizationmodulation infrared reflection-absorption spectroscopy (PM-IRRAS) ${ }^{18}$ and diffuse reflectance infrared Fourier transformed spectroscopy (DRIFTS). ${ }^{21-23}$ Its use was extended recently to X-ray absorption spectroscopy (XAS) ${ }^{24-26}$ and X-ray diffraction (XRD). ${ }^{27-29}$ Guidance for the qualitative and quantitative analysis of phase-resolved extended X-ray absorption fine structure (EXAFS) spectra was provided ${ }^{16,17}$ and demonstrated the sensitivity enhancement that is obtained. In a wider perspective, the method is applicable to any reversibly changing system and ideally without restriction to given physico-chemical methods probing the structure of the system.

In a ME experiment, a species could generally respond to a square-wave stimulation (Fig. 1a) by disappearing in one pulse and returning in the subsequent pulse, such as for example $b(t)$ and $m(t)$ in Fig. 1b. These species can be easily identified in the phase-resolved data. The identification of a short-lived species, such as $a(t)$, is less trivial. The present work

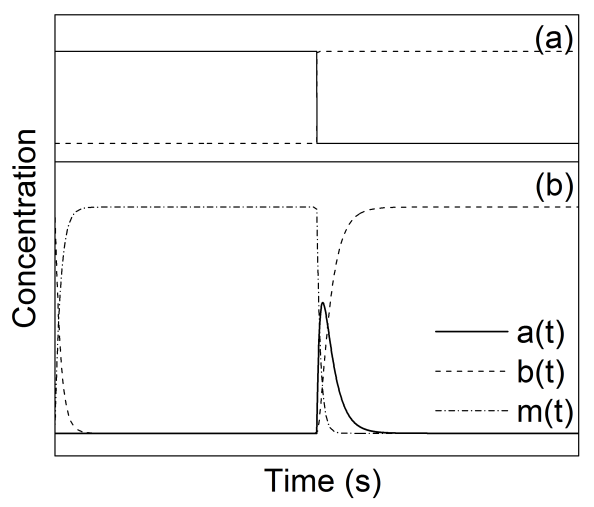

Figure 1: (a) Temporal evolution of the concentration of the inlet gases in a ME experiment applying a square-wave stimulation. (b) Possible temporal evolution of species responding to the external stimulation. $a(t)$ is a short-lived species.

demonstrates how the sensitivity to this species can be increased upon variation of either the frequency of the external stimulation $(\omega)$ or the demodulation frequency $(n \omega)$ after data acquisition at fixed $\omega$. Experimental results are provided to support our theoretical analysis. 


\section{Variation of the modulation frequency}

The present section demonstrates how the sensitivity to short-lived species increases by increasing the frequency of the external stimulation $\omega$ during the experiment.

\section{Fourier analysis of a simple exponential decay}

In electrical and electronic engineering ${ }^{30}$ a signal is the evolution of a measurable quantity as function of an independent variable. The time variable $t$ is often the independent variable. A system $O$ is a device which modifies an input signal $x(t)$ in an output signal $y(t)=O[x(t)]$. If the input signal is the linear combination of two or more signals, and the output signal is equal to the linear combination of the output signals, the system is linear:

$$
x(t)=a \cdot x_{1}(t)+b \cdot x_{2}(t) \rightarrow y(t)=O\left[a \cdot x_{1}(t)+b \cdot x_{2}(t)\right]=a \cdot O\left[x_{1}(t)\right]+b \cdot O\left[x_{2}(t)\right]
$$

The system is time invariant when the output signal does not depend on the particular time the input is applied: if the output due to the input $x(t)$ is $y(t)$, then the output due to the input $x(t-\tau)$ is $y(t-\tau), \tau$ being a generic constant. Let $O$ be a linear time-invariant (LTI) system and $y(t)=O[x(t)]$. Then the impulse response of the system is defined as $h(t)=O[\delta(t)]$, where $\delta(t)$ is the impulse signal (Sec. 1 of SI). The output of a LTI system is given by the convolution (Sec. 2 of SI) of the input signal $x(t)$ with the impulse response $h(t): y(t)=O[x(t)]=x(t) * h(t) .{ }^{30}$ The Fourier transform of the impulse response $(\mathcal{F}\{h(t)\})$ is the frequency response $H(\omega): H(\omega)=\mathcal{F}\{h(t)\}=\int_{-\infty}^{\infty} h(t) \cdot e^{-i \omega t} d t$. If the input signal is a complex quantity $x(t)=A \cdot e^{i \omega t}$, where $A$ is the amplitude and $\omega$ the frequency, the output signal will be a complex quantity with the same frequency $\omega$, but different phase and amplitude: $y(t)=B \cdot e^{i \omega t+\varphi}$. The frequency response describes how the amplitude and the phase of the output signal are modified by the system:

$$
y(t)=h(t) * x(t)=\int_{-\infty}^{\infty} h(\tau) \cdot A \cdot e^{i \omega(t-\tau)} d \tau=A \cdot e^{i \omega t} \cdot \int_{-\infty}^{\infty} h(\tau) \cdot e^{-i \omega \tau} d \tau=x(t) \cdot H(\omega)
$$


In our study, the catalytic reacting system represents the LTI system. Most real catalytic systems are non-linear, however, when a sufficiently small stimulation is used, active species respond in a linear manner. ${ }^{10,18,31}$ The variation of the external parameter and the evolution of the species that respond to the external stimulation represent the input and output signals, respectively. If we stimulate the reacting system with a single impulse $\delta(t)$, a species could respond to the stimulation for example by following a simple exponential decay of its concentration, $c(t)$ (inset of Fig. 2a):

$$
c(t)= \begin{cases}e^{-k t}, & \text { if } t \geq 0 \\ 0, & \text { if } t<0\end{cases}
$$

where $k$ is the kinetic constant. Thus, $c(t)$ is the impulse response of the catalytic system. A short-lived species possesses a fast kinetics, which correspond to high value of $k\left(k_{2}\right.$ in the inset of Fig. 2a). If the reacting system is periodically stimulated by equal and repeated pulses instead of a single event, the input could be represented by the Shah function $I I I_{T}$ (Sec. 3 of SI) with period $T$. Then the temporal evolution of the signal of the species responding to the external stimulation is a periodic function (inset of Fig. 2b), and can be obtained from the convolution of the input $I I I_{T}$ with the impulse response $c(t): s(t)=$ $c(t) * I I I_{T}(t)$. The Fourier transform of $s(t)$ allows the transition from the time domain to the frequency domain and produces the frequency-domain representation, $S(\omega)$. The convolution in the time-domain equals the point-wise product in the frequency domain: ${ }^{30}$

$$
S(\omega)=\mathcal{F}\left\{c(t) * I I I_{T}(t)\right\}=\mathcal{F}\{c(t)\} \cdot \mathcal{F}\left\{I I I_{T}(t)\right\}
$$

The Fourier transform of the Shah function is also a sampling function with period $\omega_{0}=$ $2 \pi / T:$

$$
\begin{gathered}
\mathcal{F}\left\{I I I_{T}(t)\right\}=\frac{\sqrt{2 \pi}}{T} I I I_{\frac{2 \pi}{T}}(\omega) \\
7 \\
\text { ACS Paragon Plus Environment }
\end{gathered}
$$



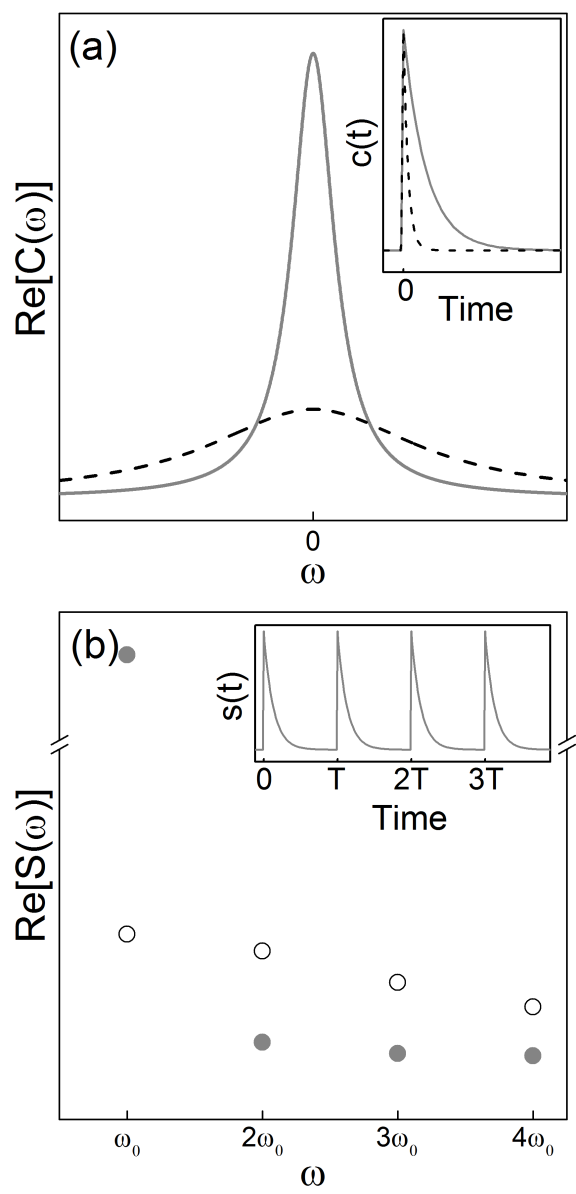

Figure 2: (a) Real part of the Fourier transform $C(\omega)$ of the concentration $c(t)$ of a generic species with simple exponential decay and different values of the kinetic constant $k$. Inset: temporal evolution of the concentration $c(t)$. (-) $k_{1},(---) k_{2}, k_{1}<k_{2}$. (b) Real part of the Fourier transform $S(\omega)$ of the concentration $s(t)$ of a generic species responding to a periodic stimulation with simple exponential decay for different values of the kinetic constant $k$. (•) $k_{1},(\circ) k_{2}, k_{1}<k_{2}$. Inset: temporal evolution of the concentration $s(t)$. 
whereas the Fourier transform of $c(t)$ is the frequency response $C(\omega)$ :

$$
C(\omega)=\int_{-\infty}^{\infty} c(t) \cdot e^{-i \omega t} d t=\int_{0}^{\infty} e^{-(k+i \omega) t} d t=\left.\frac{e^{-(k+i \omega) t}}{-(k+i \omega)}\right|_{0} ^{\infty}=\frac{k}{k^{2}+\omega^{2}}-i \frac{\omega}{k^{2}+\omega^{2}}
$$

The real part of this function, $\mathbb{R} e[C(\omega)]=\frac{k}{k^{2}+\omega^{2}}$, describes the changes in the amplitude of the output signal. $\mathbb{R} e[C(\omega)]$ is a Lorentzian function centred in 0 (Fig. 2a and Sec. 4 of $\mathrm{SI})$. The half width at half maximum is given by the kinetic constant $k$, thus the maximum amplitude is function of $k$. For low values of $k\left(k_{1}\right.$ in Fig. 2a), $\mathbb{R} e[C(\omega)]$ exhibits high amplitude for $\omega$ tending to zero, but the amplitude quickly decreases with increasing $\omega$. On the contrary, for high values of $k\left(k_{2}\right.$ in Fig. $\left.2 \mathrm{a}\right), \mathbb{R} e[C(\omega)]$ presents low amplitude at low $\omega$, but the amplitude decreases to a lower extent with increasing $\omega . S(\omega)$ is obtained by Eq. 2 and its real part is displayed in Fig. 2b. Species possessing slow kinetics ( $k_{1}$ in Fig. 2) have high intensity and therefore are easily identified at low frequency $\left(\omega_{0}\right)$. On the contrary, species with faster kinetics ( $k_{2}$ in Fig. 2) have higher intensity and are more easily identified at higher frequencies $\left(2 \omega_{0}, 3 \omega_{0}, 4 \omega_{0}\right)$. Thus, in a modulation experiment the sensitivity to short-lived species can be increased by increasing the frequency of the external stimulation $\left(\omega_{0}\right)$. In other words, decreasing the length of the period of the external stimulation $(T)$ improves our perception of transient species.

\section{Fourier analysis of experimental data}

In order to verify for a real reacting system, that the sensitivity towards transient species is improved by increasing $\omega$, modulation experiments were performed by admitting alternately $\mathrm{CH}_{4} / \mathrm{CO} / \mathrm{NO}$ and $\mathrm{O}_{2}$ on the $\mathrm{Pd} / \mathrm{Al}_{2} \mathrm{O}_{3}$ catalyst using modulation periods $T$ of $120 \mathrm{~s}, 60$ s, and $30 \mathrm{~s}$ while collecting time-resolved X-ray diffraction (XRD) patterns (see Sec. 14 of SI). The result of the following Fourier analysis does not depend on the employed method, i.e. XRD, and remains valid for other spectroscopic techniques. It should be reminded here that, since the XRD intensity is the square of the diffracted energy, the use of $2 \omega$ 
$(n=2)$ is required to detect periodically changing signals of active species in phase-resolved XRD patterns. ${ }^{27}$ We present here the data for $n=1$ because we did not observe any difference between the two demodulation frequencies. Fig. 3a-b display the time-resolved
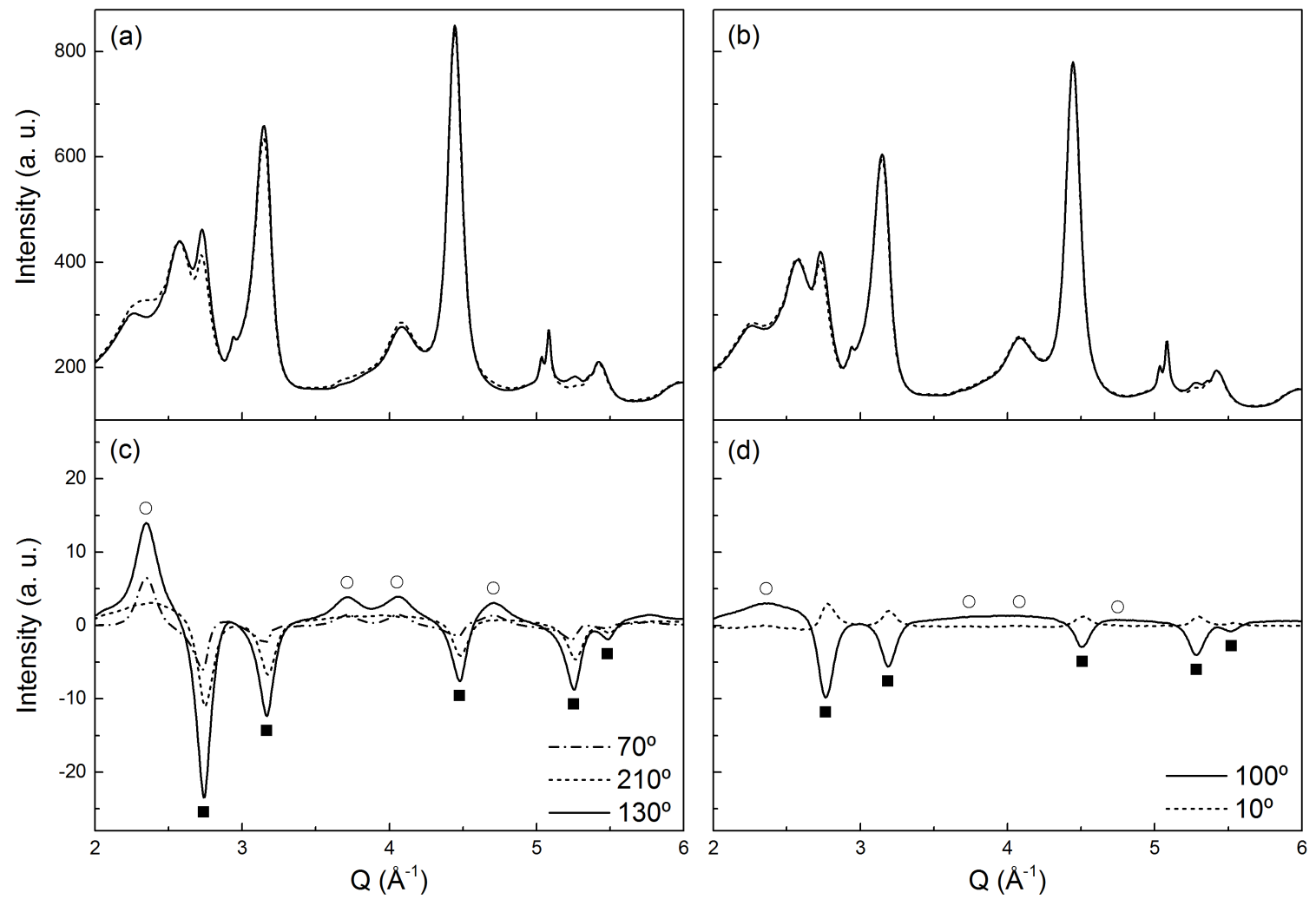

Figure 3: Averaged time-resolved XRD patterns recorded at the end of the pulses (-) in $0.25 \mathrm{vol} \% \mathrm{CH}_{4} / 1 \mathrm{vol} \% \mathrm{CO} / 0.22 \mathrm{vol} \% \mathrm{NO} / \mathrm{He}$ and $(---)$ in $1 \mathrm{vol} \% \mathrm{O}_{2} / \mathrm{He}$ during the modulation experiments at $320^{\circ} \mathrm{C}$ on 1.6 wt. $\% \mathrm{Pd} / \gamma-\mathrm{Al}_{2} \mathrm{O}_{3}$ with modulation period of (a) $120 \mathrm{~s}$ and (b) $30 \mathrm{~s}$. (c - d) Selected phase-resolved XRD patterns. (a) Pd, (o) PdO.

XRD patterns obtained using a modulation period of $120 \mathrm{~s}$ and $30 \mathrm{~s}$, respectively. The patterns are dominated by the reflections of $\gamma-\mathrm{Al}_{2} \mathrm{O}_{3}$. Small intensity differences can be observed at ca. $2.35,2.74,3.16,5.30 \AA^{-1}$ that are attributed to the periodic reduction and oxidation of palladium. Changes in the time resolved patterns are more evident in the case of a longer modulation period in Fig. 3a. The sensitivity towards subtle structural changes that may not be visible by inspection of the time-resolved data is enhanced using phase sensitive detection (PSD). ${ }^{25,29}$ First, PSD removes the contribution of alumina, because it does not respond to the concentration modulation. Fig. 3c-d report selected phase-resolved 
patterns, which highlight the reflections that experience intensity changes and facilitate the identification of their position.

The reflections at ca. $2.35,2.78,3.21$ and $5.31 \AA^{-1}$ correspond to the small variations in the intensity observed in the time-resolved patterns. In addition, the intensity change of the reflections at ca. $3.74,4.08,4.52,4.75$, and $5.54 \AA^{-1}$ becomes clearly visible only in the PSD data. The signals at $2.78,3.21,4.52,5.31$, and $5.54 \AA^{-1}$ (corresponding to $2 \theta=$ $39.9^{\circ}, 46.4^{\circ}, 67.3^{\circ}, 81.2^{\circ}$, and $85.6^{\circ}$ for $\mathrm{Cu} \mathrm{K \alpha}$ radiation) are associated with the formation of the metallic palladium phase, ${ }^{25,29,32}$ while the reflections at ca. $2.35,3.74,4.08$, and 4.75 $\AA^{-1}\left(33.5^{\circ}, 54.6^{\circ}, 60^{\circ}\right.$, and $\left.71.2^{\circ}\right)$ to the formation of the PdO phase ${ }^{25,29}$ upon re-oxidation. The phase-resolved signals reach the maximum amplitude in the in-phase patterns. ${ }^{20}$ These were obtained at $\phi^{P S D}=130^{\circ}$ and $100^{\circ}$ when the modulation period was $120 \mathrm{~s}$ and $30 \mathrm{~s}$, respectively. The peaks of metallic $\mathrm{Pd}$ in the in-phase patterns in the two experiments have different intensity, but always exhibit a sharp and defined shape, indicating that metallic $\mathrm{Pd}$ is present as a crystalline phase. In contrast, it is evident that the reflections of $\mathrm{PdO}$ present different shapes in the two experiments. When using a long modulation period, they exhibit a complex shape (Fig. 3c) that clearly derives from the sum of two contributions. The two contributions can be isolated by careful analysis of the phase angular dependence of the XRD signals. ${ }^{15-17,33}$ The patterns obtained with $\phi^{P S D}$ equal to $210^{\circ}$ and $70^{\circ}$ are able to isolate the two contributions. The reflections of $\mathrm{PdO}$ are not well defined in the phaseresolved pattern obtained for $\phi^{P S D}=210^{\circ}$. The reflection at ca. $2.35 \AA^{-1}$ is broad, while a single broad feature covers the region where two reflections should appear at 3.74 and $4.08 \AA^{-1}$. From a XRD perspective, this line shape suggests the formation of a disordered phase, that we define as amorphous $\mathrm{PdO}\left(P d O_{a}\right)$ for simplicity. This may correspond to some form of surface oxide layer on reduced $\mathrm{Pd}$. The $\mathrm{PdO}$ reflections exhibit a more defined shape at $\phi^{P S D}=70^{\circ}$, indicating the formation of an ordered phase (crystalline $\mathrm{PdO}, P d O_{c}$ ). Because signals possessing fast kinetics appear at higher $\phi^{P S D}$ in the phase-domain (Sec. 5 of SI), the broad and undefined shape at $\phi^{P S D}=210^{\circ}$ indicates the initial fast formation of 
$P d O_{a}$, whereas the sharp peaks visible at $\phi^{P S D}=70^{\circ}$ reveal the subsequent slower evolution into $P d O_{c}$. The second stage of the oxidation proceeds more slowly because the formation of the crystalline phase requires massive restructuring ${ }^{34}$ and diffusion limitation can occur due to the surface oxide. The reflections attributed to the formation of the $\mathrm{PdO}$ phase in the in-phase pattern obtained at $100^{\circ}$ with the shortest modulation period (30 s, Fig. 3d) equal the ones in the phase-resolved pattern obtained at $\phi^{P S D}=210^{\circ}$ when using a longer period of modulation (120 s, Fig. 3c). This result confirms that $P d O_{a}$ is an intermediate species in the re-oxidation process. When the modulation period is reduced to $30 \mathrm{~s}$ there is not enough time for the formation of $\mathrm{PdO}_{c}$ and the features of $\mathrm{PdO}$ can be more easily identified. Only upon longer exposure to $\mathrm{O}_{2}$, bulk oxidation proceeds with the formation of a crystalline $\mathrm{PdO}$ phase. The formation of an amorphous surface oxide on metallic $\mathrm{Pd}$ prior to the onset of the bulk oxidation was previously observed on $\mathrm{Pd}$ single crystals, ${ }^{34,35}$ on $\mathrm{SiO}_{x^{-}}$ supported Pd nanoparticles of 15-35 nm, ${ }^{35}$ and on Pd particles of ca. $20 \mathrm{~nm}^{36}$ supported on $\theta$-alumina by in situ surface $\mathrm{XRD}^{34}$ and X-ray photoelectron spectroscopy (XPS). ${ }^{35,36}$ It is important to note here that the results of the present study were obtained with a powder sample containing low levels of Pd and nanoparticles of 2-5 $\mathrm{nm}$.

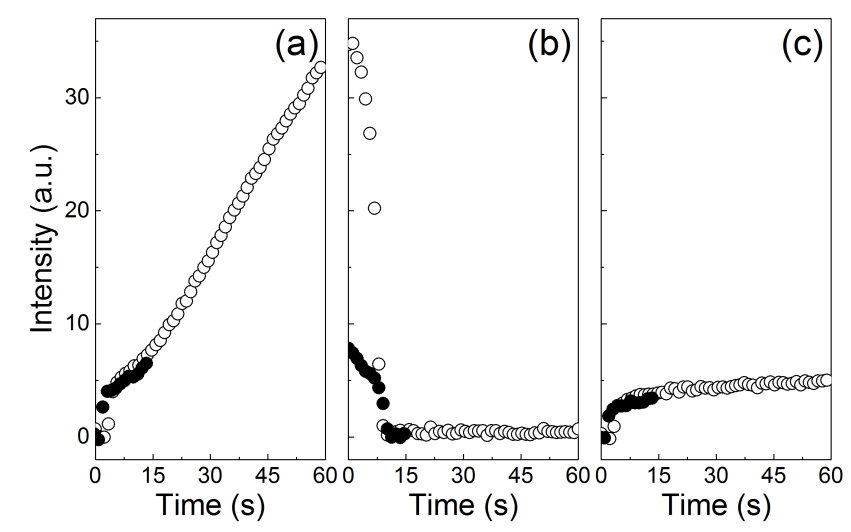

Figure 4: Temporal evolution of $\mathrm{PdO}(002)\left(2.35 \AA^{-1}\right)$ during (a) 1 vol\% $\mathrm{O}_{2} / \mathrm{He}$ and (b) 0.25 vol\% $\mathrm{CH}_{4} / 1 \mathrm{vol} \% \mathrm{CO} / 0.22 \mathrm{vol} \% \mathrm{NO} / \mathrm{He}$ pulses. (c) Temporal evolution of the amorphous $\mathrm{PdO}$ phase $\left(2.10 \AA^{-1}\right)$ during the 1 vol $\% \mathrm{O}_{2} / \mathrm{He}$ pulse. Modulation period of (o) $120 \mathrm{~s}$ and (•) $30 \mathrm{~s}$.

Fig. 4a-b displays the temporal profile of the $\mathrm{PdO}(002)$ reflection $\left(2.35 \AA^{-1}\right)$. When the 

delay of ca. 2-3 s upon $\mathrm{O}_{2}$ admittance (Fig. 4a); then the rate of signal increase changed and the evolution proceeded more slowly. We attribute the initial fast increase to the formation of $P d O_{a}$, and the subsequent slower evolution to bulk oxidation and formation of $P d O_{c}$. $P d O_{a}$ can be isolated by selecting the signal at ca. $2.10 \AA^{-1}$ that does not contribute to that of $P d O_{c}$. Fig. 4c reports its temporal profile in the $\mathrm{O}_{2}$ pulse. In the first ca. $5 \mathrm{~s}$ the evolution is similar to that of the $\mathrm{PdO}(002)$ reflection, then the signal is stable and does not further increases. The formation of $P d O_{a}$ produces a small XRD signal increase, whereas a much higher intensity gain derives from the formation of $P d O_{c}$ in agreement with the sensitivity of XRD towards crystalline phases. Only the combination with PSD allows the identification of $P d O_{a}$. The initial delay in $\mathrm{PdO}$ formation is probably due to the oxidation of the adsorbed species formed on the catalytic surface during the reducing pulse in $\mathrm{CH}_{4} / \mathrm{CO} / \mathrm{NO}$. The subsequent reduction is completed in ca. $10 \mathrm{~s}$ (Fig. 4b). When the modulation period was reduced to $30 \mathrm{~s}$, the formation of the amorphous oxide occurred immediately at the $\mathrm{CH}_{4} / \mathrm{CO} / \mathrm{NO} \rightarrow \mathrm{O}_{2}$ switch. As in the experiment with longer modulation period, after ca. $3 \mathrm{~s}$ the evolution of $\mathrm{PdO}$ slows down and the subsequent reduction is completed in ca. 10 s. The short (15 s) reducing pulse is enough for PdO reduction, but probably less adsorbed species are formed on the catalytic surface, thus tentatively explaining the absence of the delay at the beginning of the $\mathrm{O}_{2}$ pulse.

The Fourier analysis is applied to the oxidising pulse with the aim to demonstrate that the sensitivity to intermediate species possessing fast kinetics and having transient appearance can be increased by using higher frequency of modulation $(\omega)$. From the analysis of the experimental data of Fig. 3, we have proposed that during the $\mathrm{O}_{2}$ pulse the formation of $P d O_{a}$ occurs initially due to $\mathrm{O}_{2}$ dissociative adsorption on reduced $\mathrm{Pd}$. For simplicity, we consider that one oxygen atom reacts with one $\mathrm{Pd}$ atom and that all adsorption sites are equivalent and the adsorption not dependent on coverage (Langmuir model). The surface coverage is assumed to be low, thus $\mathrm{O}_{2}$ desorption is neglected. Then the slow transformation 
of $P d O_{a}$ into $P d O_{c}$ takes place:

$$
\begin{gathered}
P d+\frac{1}{2} O_{2} \stackrel{k_{1}}{\rightarrow} P d O_{a} \\
P d O_{a} \stackrel{k_{2}}{\longrightarrow} P d O_{c}
\end{gathered}
$$

The first step of oxidation is fast, while the second step is slower (Fig. 4a); and $k_{1}>k_{2}$. In the present case, $P d O_{a}$ is the transient species. If $m(t), a(t)$, and $b(t)$ are the concentrations of $\mathrm{Pd}, \mathrm{PdO}_{a}$, and $\mathrm{PdO}_{c}$, respectively, their variation in the $\mathrm{O}_{2}$ pulse is

$$
\begin{aligned}
& \frac{d[m(t)]}{d t}=-k_{1} \cdot P_{O_{2}}^{1 / 2} \cdot m(t)=-C_{1} \cdot m(t) \\
& \frac{d[a(t)]}{d t}=k_{1} \cdot P_{O_{2}}^{1 / 2} \cdot m(t)-k_{2} \cdot a(t)=C_{1} \cdot m(t)-k_{2} \cdot a(t) \\
& \frac{d[b(t)]}{d t}=k_{2} \cdot a(t)
\end{aligned}
$$

Under the hypothesis of a differential reactor, the concentration of the reactant gases at the inlet and at the outlet of the reactor is approximately the same and constant. Therefore, $P_{O_{2}}$ is constant, and the constant $C_{1}=k_{1} \cdot P_{O_{2}}^{1 / 2}$ can be defined. The assumption of the differential reactor is a simplification, but it is reasonable since the residence time (ca. 0.03 $\mathrm{s}$ ) is much lower than the duration of a single pulse (60 s or $15 \mathrm{~s}$ ) and the breakthrough of $\mathrm{O}_{2}$ (Fig. S6) occurs immediately after the switch.

The temporal evolution of $m(t), a(t)$, and $b(t)$ during the $\mathrm{O}_{2}$ pulse is obtained solving the differential equations (Sec. 6 of SI):

$$
\begin{aligned}
& m(t)=e^{-C_{1} \cdot t} \\
& a(t)=\frac{C_{1}}{k_{2}-C_{1}} \cdot\left(e^{-C_{1} \cdot t}-e^{-k_{2} \cdot t}\right) \\
& b(t)=\frac{C_{1}}{k_{2}-C_{1}} \cdot e^{-k_{2} \cdot t}-\frac{k_{2}}{k_{2}-C_{1}} \cdot e^{-C_{1} \cdot t}+1
\end{aligned}
$$

The temporal profiles are displayed in Fig. 5a. Clearly the profiles of $a(t)+b(t)$ and $a(t)$ 
do not coincide with the temporal evolution of $\mathrm{PdO}(002)$ (Fig. 4a) and $\mathrm{PdO}_{a}$ (Fig. 4c), respectively. The inconsistency has likely two sources. First, the formation of $P d O_{c}$ produces high signal increase because XRD requires long-range order and is sensitive to crystalline phases. In contrast, the evolution of $\mathrm{PdO}_{a}$ produces a much smaller intensity gain because it is difficult to observe the amorphous phases. Therefore, $a(t)$ and $b(t)$ were multiplied for two constants, $C_{a}$ and $C_{b}$, respectively, with $C_{a}<<C_{b}$, in order to rescale the temporal evolution of concentration of the two phases (Fig. 5a) with respect to that of the XRD signals (Fig. 4). In addition, also the time-scale is not comparable. Because $k_{2}<<k_{1}$, the evolution of the XRD signals of $P d O_{a}$ and $P d O_{c}$ in Fig. 4 represents only a small initial part of the kinetic of Fig. 5a. The inset of Fig. 5a displays the initial evolution of $a(t) \cdot C_{a}$, $b(t) \cdot C_{b}$, and their sum. These scaled temporal profiles become qualitatively similar to the evolution of the XRD signals of Fig. 4a and c.

The temporal profiles are necessary for the following Fourier analysis. The frequency responses $A(\omega)$ and $B(\omega)$ can be obtained through the Fourier transform of $a(t)$ and $b(t)$ (Sec. 7 of SI):

$$
\begin{aligned}
& A(\omega)=\frac{C_{1}}{k_{2}-C_{1}} \cdot\left(\frac{C_{1}-i \omega}{C_{1}^{2}+\omega^{2}}-\frac{k_{2}-i \omega}{k_{2}^{2}+\omega^{2}}\right) \\
& B(\omega)=\frac{C_{1}}{k_{2}-C_{1}} \cdot \frac{k_{2}-i \omega}{k_{2}^{2}+\omega^{2}}-\frac{k_{2}}{k_{2}-C_{1}} \cdot \frac{C_{1}-i \omega}{C_{1}^{2}+\omega^{2}}-\frac{i}{\omega^{2}}
\end{aligned}
$$

The real part of the frequency response describes the changes in the amplitude of $a(t)$ and $b(t)$ due to the external stimulation. Therefore, it should suggest which value of $\omega$ permits more easily the identification of one or the other species. The real parts of the frequency responses are:

$$
\begin{aligned}
& \mathbb{R e}[A(\omega)]=\frac{C_{1}}{k_{2}-C_{1}} \cdot\left(\frac{C_{1}}{C_{1}^{2}+\omega^{2}}-\frac{k_{2}}{k_{2}^{2}+\omega^{2}}\right) \\
& \mathbb{R e}[B(\omega)]=\frac{C_{1}}{k_{2}-C_{1}} \cdot \frac{k_{2}}{k_{2}^{2}+\omega^{2}}-\frac{k_{2}}{k_{2}-C_{1}} \cdot \frac{C_{1}}{C_{1}^{2}+\omega^{2}}
\end{aligned}
$$


Figure 5: (a) Temporal evolution of metallic $\mathrm{Pd}(m(t),-\cdot-)$, amorphous surface oxide $(a(t)$, -), crystalline bulk PdO $(b(t),---)$, and $a(t)+b(t)(-)$ during the $\mathrm{O}_{2}$ pulse. Inset: initial evolution of $(-) a(t) \cdot C_{a},(---) b(t) \cdot C_{b},(-) a(t) \cdot C_{a}+b(t) \cdot C_{b}$, with $C_{a}, C_{b}$ constants, and $C_{a}<<C_{b}$. (b) Real part of the frequency response of the amorphous surface oxide $(\mathbb{R} e[A(\omega)]$, $-)$ and of the crystalline bulk PdO $(\mathbb{R e} e[B(\omega)],---) . \omega_{0}^{2}=C_{1} k_{2} ; \omega_{1}^{2}=k_{2}\left(2 C_{1}+k_{2}\right)$.
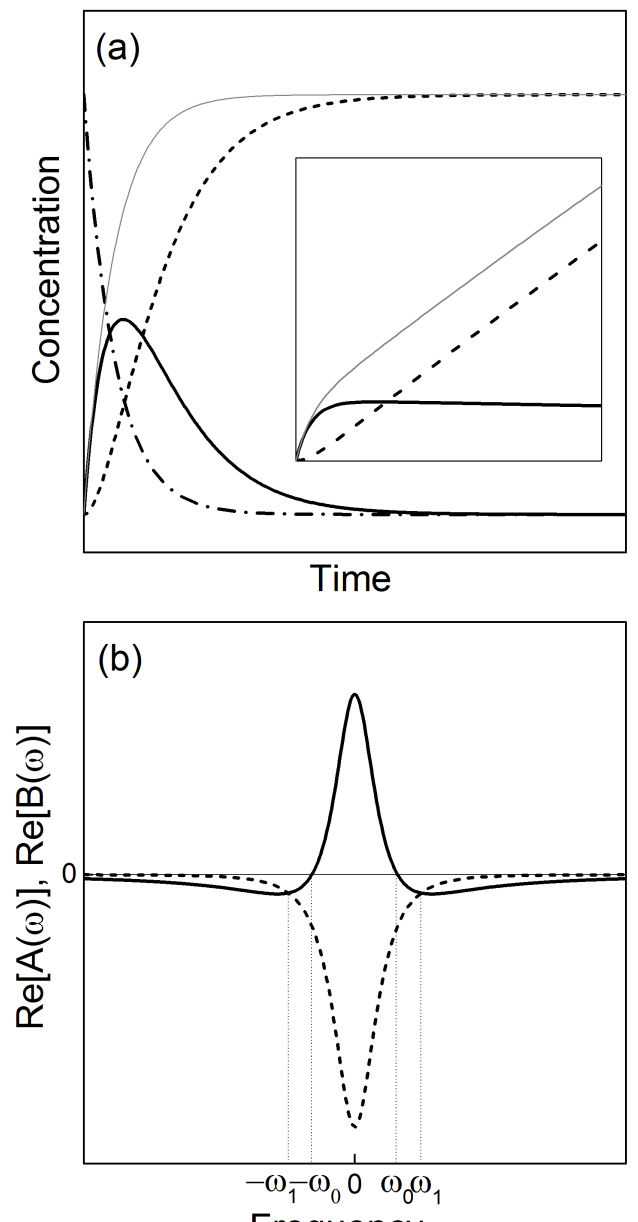

Frequency $\omega$ 
They are differences of two Lorentzian functions and are displayed in Fig. 5b. Because our aim is to find the value of $\omega$ that facilitates the identification of the intermediate species, i.e. $P d O_{a}$, we need to find under which conditions the absolute intensity of $\mathbb{R} e[A(\omega)]$ is higher than the absolute intensity of $\mathbb{R} e[B(\omega)]$ :

$$
|\mathbb{R} e[A(\omega)]|>|\mathbb{R} e[B(\omega)]|
$$

The solution of Eq. 6 is reported in Sec. 8 of SI. In summary, at low modulation frequency $\left(\omega^{2}<C_{1} k_{2}\right)$, the real part of the frequency response of the amorphous oxide $\mathbb{R} e[A(\omega)]$ exhibits lower intensity compared to the real part of the frequency response of the bulk oxide $\mathbb{R} e[B(\omega)]$ (Fig. 5b). Therefore, the phase-resolved signal of the amorphous oxide exhibits lower intensity compared to the phase-resolved signal of the bulk oxide. The experiment performed with a modulation period of $120 \mathrm{~s}$ corresponds to this case. If the signals overlap, such as in the case of the XRD experiment for $P d O_{a}$ and $P d O_{c}$, the identification and isolation of the transient species is more difficult. The formation of $\operatorname{Pd} O_{a}$ was in fact not clear only by observing the in-phase pattern obtained with $T=120 \mathrm{~s}$ (Fig. 3c, 130 $)$. In this situation, the careful selection of phase-resolved signals is of help. Since the frequency responses of the two species have opposite sign (Fig. $5 \mathrm{~b}, \mathbb{R} e[A(\omega)]>0$ and $\mathbb{R} e[B(\omega)]<0)$, the phase-resolved signals exhibited different phase-lag. Thus, the amorphous phase could be isolated and identified at $\phi^{P S D}=210^{\circ}$ (Fig. 3c).

At high modulation frequency $\left(\omega^{2}>C_{1} k_{2}\right), a(t)$ (amorphous $\mathrm{PdO}$ ) and $b(t)$ (crystalline $\mathrm{PdO}$ ) will be in phase in the frequency domain. Indeed the real parts of the frequency response are both negative (Fig. 5b). The signals of the amorphous phase will have higher intensity and therefore will be more easily identified at higher frequency, i.e. if $\omega^{2}>k_{2}\left(2 C_{1}+\right.$ $k_{2}$ ). In agreement, $P d O_{a}$ was immediately visible in the phase-resolved pattern obtained with shorter modulation period, i.e. $30 \mathrm{~s}$ (Fig. 3d, 100).

Thus, we have demonstrated that by increasing the frequency of the external modulation 
$\omega$ the isolation and identification of a transient species with faster kinetics is facilitated.

\section{Variation of the demodulation frequency}

In the previous section we have demonstrated that the variation of the frequency of modulation $\omega$ can increase the sensitivity of an in situ characterization method. The use of a square-wave stimulation provides similar information to that of several experiments with sinusoidal-wave stimulations at the same frequency of the square-wave stimulation and at higher frequencies. ${ }^{18}$ Therefore, another strategy to increase the sensitivity towards shortlived species could be the application of the square wave stimulation with frequency $\omega$ during the measurement, and after the collection of time-resolved data, the variation of the demodulation frequencies $n \omega$ during the data analysis. In a previous study ${ }^{23}$ we provided empirical evidence that transient species can be in principle discerned with improved sensitivity in the phase-resolved data obtained with higher demodulation frequency $n \omega$, in other words with $n>1$. The variation of the demodulation frequency allowed the identification of gaseous $\mathrm{CO}_{2}$ and of isocyanate species adsorbed on $\mathrm{Rh}$ in the phase-resolved DRIFT spectra collected during $\mathrm{CO}$ and $\mathrm{NO}$ pulses on $\mathrm{Rh} / \mathrm{Al}_{2} \mathrm{O}_{3}$ catalyst (Sec. 9 of SI). Because PSD with $n>1$ is not standard, this section investigates this topic from a more rigorous mathematical perspective.

As in the previous section, in order to simplify the mathematics, we consider two generic species whose concentrations respond to the external stimulation with a simple exponential decay (inset of Fig. 2a): $c_{1}(t)=e^{-k_{1} t}$ and $c_{2}(t)=e^{-k_{2} t}$, where $k_{1}$ and $k_{2}$ are the kinetic constants, are positive and $k_{1}<k_{2}$. The simple exponential decay function can represent the temporal evolution of a transient species that appears only in one pulse (i.e. $a(t)$ in Fig. 1b). The increase of $k$ shortens the life of the species, thus its identification becomes more difficult. When PSD is applied, the corresponding phase-resolved signals are obtained using 
Eq. 1 (Sec. 10 of SI):

$$
c_{i, n}\left(\phi^{P S D}\right)=\frac{2}{T} \cdot \frac{1-e^{-k_{i} T}}{k_{i}^{2}+n^{2} \cdot \omega^{2}} \cdot\left[k_{i} \cdot \sin \left(\phi^{P S D}\right)+n \omega \cdot \cos \left(\phi^{P S D}\right)\right]
$$

Fig. 6 compares the corresponding phase angular dependence obtained by increasing the demodulation frequency $n \omega$. As shown in Fig. 6a, the intensity of the phase-resolved signal $c_{n=1}\left(\phi^{P S D}\right)$ is lower for larger $k\left(k_{2}\right)$ when $n=1$. The absolute intensity of the phase-resolved signals decreases with increasing the value of $n$ (Fig. 6b-e). However, the intensity of the signal corresponding to the species with slower kinetics $\left(k_{1}\right)$ is attenuated more than that of the species with faster kinetics $\left(k_{2}\right)$. Thus, the ratio $R$ between the maximum values of the

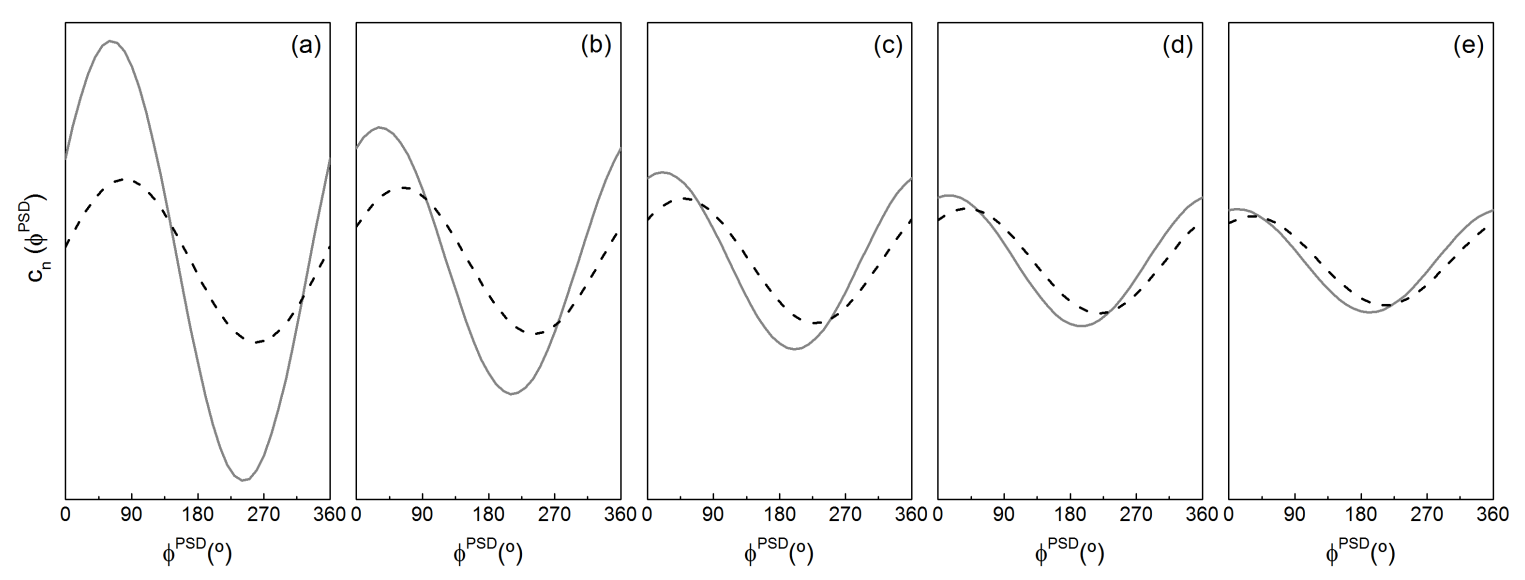

Figure 6: Phase angular dependence of the signals of two generic species responding to the external stimulation with a simple exponential decay obtained with (a) $n=1$, (b) $n=3$, (c) $n=5$, (d) $n=7$, (e) $n=9$. (-) $k_{1},(---) k_{2}, k_{1}<k_{2}$.

phase-resolved signals:

$$
R=\frac{\max \left[c_{1, n}\left(\phi^{P S D}\right)\right]}{\max \left[c_{2, n}\left(\phi^{P S D}\right)\right]}=\frac{C_{1, n}}{C_{2, n}}
$$

should decrease with increasing $n$. If $R$ decreases when $n$ increases, the partial derivative of $R$ with respect to $n$ is negative:

$$
\frac{\delta(R)}{\delta n}<0
$$

In Eq. $7, \sin \left(\phi^{P S D}\right)$ and $\cos \left(\phi^{P S D}\right)$ vary between \pm 1 , therefore in first approximation the 
maximum value of the phase resolved signals could be:

$$
C_{i, n} \sim \frac{2}{T} \cdot \frac{1-e^{-k_{i} T}}{k_{i}^{2}+n^{2} \cdot \omega^{2}} \cdot\left(k_{i}+n \omega\right)
$$

In Eq. 10, the term $1-e^{-k_{i} T}$ is positive for positive values of $k_{i}$, and increases with increasing $k_{i}$, but it tends asymptotically to 1 . The denominator $k_{i}^{2}+n^{2} \cdot \omega^{2}$ is a second power function of $k_{i}$, and increases its value much faster than the term $1-e^{-k_{i} T}$. Therefore, the term $1-e^{-k_{i} T}$ can be neglected, affording:

$$
C_{i, n} \sim \frac{2}{T} \cdot \frac{k_{i}+n \omega}{k_{i}^{2}+n^{2} \cdot \omega^{2}}
$$

At this point, three different cases can be distinguished:

1. $n \omega<<k_{1}, k_{2}$ : the demodulation frequency is much smaller than the reaction rate. Considering that the demodulation frequency is equal or multiple of the modulation frequency ( $n$ can only assume odd integer positive values ${ }^{18}$ ), the frequency of modulation $\omega$ is smaller than the reaction rate. By the modulation approach the system should be stimulated, if the stimulation frequency is too low in comparison to the reaction rate the system would be in stationary conditions. Consequently this first case is not very significant. Nevertheless, $n \omega$ in the numerator of Eq. 11 can be neglected, affording: $C_{i, n} \sim \frac{2}{T} \cdot \frac{k_{i}}{k_{i}^{2}+n^{2} \cdot \omega^{2}}$. Thus, Eq. 8 becomes:

$$
R=\frac{k_{1} \cdot\left(k_{2}^{2}+n^{2} \cdot \omega^{2}\right)}{k_{2} \cdot\left(k_{1}^{2}+n^{2} \cdot \omega^{2}\right)}
$$

and its partial derivative with respect to $n$ (Sec. 11 of $\mathrm{SI}$ ) is:

$$
\frac{\delta(R)}{\delta n}=\frac{2 \cdot k_{1} \cdot k_{2} \cdot n \cdot \omega^{2} \cdot\left(k_{1}^{2}-k_{2}^{2}\right)}{\left[k_{2} \cdot\left(k_{1}^{2}+n^{2} \cdot \omega^{2}\right)\right]^{2}}
$$

The denominator of Eq. 13 is always positive. In the numerator, the term $2 \cdot k_{1} \cdot k_{2} \cdot n \cdot \omega^{2}$ 
is always positive, whereas the term $k_{1}^{2}-k_{2}^{2}$ is always negative for $k_{1}<k_{2}$. Therefore, Eq. 9 is always verified.

2. $n \omega \sim k_{1}$ and $n \omega<<k_{2}$ : the demodulation frequency is smaller than the kinetic constant of the fast reaction, but it has the same magnitude of that of the slow reaction. Therefore, the maximum values of the phase-resolved signals approximated by Eq. 11 become: $C_{1, n} \sim \frac{2}{T} \cdot \frac{2 k_{1}}{k_{1}^{2}+n^{2} \cdot \omega^{2}}$, and $C_{2, n} \sim \frac{2}{T} \cdot \frac{k_{2}}{k_{2}^{2}+n^{2} \cdot \omega^{2}}$. The ratio $R$ (Eq. 8) becomes:

$$
R=2 \cdot \frac{k_{1} \cdot\left(k_{2}^{2}+n^{2} \cdot \omega^{2}\right)}{k_{2} \cdot\left(k_{1}^{2}+n^{2} \cdot \omega^{2}\right)}
$$

that is twice the value of $R$ obtained in the first case (Eq. 12). As a consequence, also the partial derivative of $R$ with respect to $n$ is twice the value obtained in the first case (Eq. 13 and Sec. 11 of SI):

$$
\frac{\delta(R)}{\delta n}=4 \cdot \frac{k_{1} \cdot k_{2} \cdot n \cdot \omega^{2} \cdot\left(k_{1}^{2}-k_{2}^{2}\right)}{\left[k_{2} \cdot\left(k_{1}^{2}+n^{2} \cdot \omega^{2}\right)\right]^{2}}
$$

Also in this case, Eq. 9 is always verified for $k_{1}<k_{2}$.

3. $n \omega>>k_{1}, k_{2}$ : the frequency of demodulation is much larger than the rate of the two reactions. Therefore, $k_{i}$ can be neglected in the numerator of Eq. 11, affording: $C_{i, n} \sim \frac{2}{T} \cdot \frac{n \cdot \omega}{k_{i}^{2}+n^{2} \cdot \omega^{2}}$, and the ratio $R$ (Eq. 8) becomes:

$$
R=\frac{k_{2}^{2}+n^{2} \cdot \omega^{2}}{k_{1}^{2}+n^{2} \cdot \omega^{2}}
$$

while its partial derivative with respect to $n$ is (Sec. 11 of $\mathrm{SI}$ ):

$$
\frac{\delta(R)}{\delta n}=\frac{2 \cdot n \cdot \omega^{2} \cdot\left(k_{1}^{2}-k_{2}^{2}\right)}{\left(k_{1}^{2}+n^{2} \cdot \omega^{2}\right)^{2}}
$$

As in the previous cases, Eq. 16 is always negative for $k_{1}<k_{2}$.

Therefore, Eq. 9 is always verified for $k_{1}<k_{2}$. We have demonstrated that the intensity 
of the phase-resolved signal corresponding to species with low kinetic constant $\left(k_{1}\right)$ is more attenuated than species possessing fast kinetics $\left(k_{2}\right)$. Therefore, the contribution of species with fast kinetics, thus shorter lifetime, is enhanced for $n>1$ relative to species with lower $k$ and can be in principle discerned with enhanced sensitivity by applying higher frequency of demodulation ( $n \omega$ with $n>1$ ). The advantage is even more evident if the phase angular dependence of a transient species, i.e. $a(t)$ in Fig. $1 \mathrm{~b}$ and $a\left(\phi^{P S D}\right)$ in Fig. S4, is compared with those of the species that reach a steady state in one of the semi-periods, i.e. $b(t)$ and $m(t)$. The recognition of the short-lived species is difficult with $n=1$ because of the lower intensity of its signal, whereas is facilitated by increasing $n$. However, it should be noted in Eq. 11 that the maximum intensity of the phase-resolved signal is directly proportional to $k_{i}$, but inversely proportional to the square of $k_{i}$. Thus, $C_{1, n}$ is always higher than $C_{2, n}$ for $k_{1}<k_{2}$, or at the limit equal to $C_{2, n}$ for very high value of $n$ (Fig. 6 and Sec. 13 of SI). An upper limit exists for the exploitation of the demodulation frequency that depends on the quality of the time-resolved data set. Since the amplitude of the phase-resolved signal is inversely proportional to $n$, the signal-to-noise ratio of the PSD data decreases by increasing $n$. The signal intensities are also not always proportional to the species concentrations. For instance, the crystallinity and the absorption cross-section influence the detected XRD and spectroscopic signals, respectively. Therefore the use of higher demodulation frequencies and $n>1$ can help to discern different species, but it should be applied with care. Recalling the previous time-resolved XRD example (Fig. 3a), after data treatment by PSD with higher demodulation frequencies, i.e. $n=3,5,9$ in Eq. 1 (Fig. S5), the increase of the demodulation frequency does not facilitate the identification of the two $\mathrm{PdO}$ contributions. The difficulty in the identification of $\mathrm{PdO}$ is mainly due to the low sensitivity of XRD towards disordered phases. Since its formation is faster than that of $\mathrm{PdO} O_{c}$, the increase of $\omega$ allows its easier recognition, but the increase of the demodulation frequency does not help. 


\section{Conclusions}

The present work investigated how to increase the sensitivity to species possessing fast kinetics when combining the modulation approach with phase sensitive detection (PSD). Two different strategies were considered.

First, given a system responding to a periodic stimulation with a simple exponential decay, we demonstrated using the Fourier analysis that the increase of $\omega$, or in other words the decrease of the modulation period $T$, enhances the sensitivity to short-lived species thus possessing fast kinetics. This was verified for a real system, where the species exhibit different temporal profiles. For this purpose, time-resolved XRD patterns were collected on 1.6 wt.\% $\mathrm{Pd} / \gamma-\mathrm{Al}_{2} \mathrm{O}_{3}$ during alternate pulses of $\mathrm{CH}_{4} / \mathrm{CO} / \mathrm{NO}$ and $\mathrm{O}_{2}$ at $320^{\circ} \mathrm{C}$ employing modulation periods $T$ of different length. The analysis of the phase-resolved patterns suggested that the Pd oxidation occurred in two stages. The initially quick formation of a disordered oxide phase $\left(P d O_{a}\right)$ was followed by a slower evolution into a crystalline oxide phase $\left(P d O_{c}\right)$. Despite the overlap, the signature of $\mathrm{PdO}_{a}$ could be isolated in the phase-resolved data. At higher frequency the signal of $\operatorname{PdO}_{a}$ exhibited higher intensity than that of $P d O_{c}$, and was thus more easily identified.

An alternative approach to performing many experiments with different $\omega$, is the use of higher demodulation frequencies $(n \omega, n>1)$ during data analysis. The absolute intensity of the phase-resolved signals is reduced by increasing $n$. However, the signals of transient species with fast kinetics are less attenuated than those of other species. Thus, the former species are relatively enhanced and can be in principle discerned with improved sensitivity in the phase-resolved data obtained with $n>1$. In a practical example, gas phase $\mathrm{CO}_{2}$ and isocyanate on Rh could be identified in the phase-resolved infrared spectra of 2 wt\% $\mathrm{Rh} / \gamma-\mathrm{Al}_{2} \mathrm{O}_{3} \cdot{ }^{23}$ On the other hand, the application of higher $n \omega$ is not always advantageous and did not facilitate the identification of $P d O_{a}$ in the XRD patterns collected with the long modulation period.

The advantage of the variation of the demodulation frequency $(n)$ over the modulation 
frequency $(\omega)$ is that only one modulation experiment is necessary. This may become especially convenient in the case of synchrotron based techniques, where the measuring time is limited and precious. In addition, the catalytic system may change irreversibly in the case of very long $T$ and the results of the experiment may be not comparable with the ones obtained in a previous measurement employing shorter $T$. Thus, caution is needed when comparing experiments performed with different $\omega$.

\section{Acknowledgement}

The authors kindly acknowledge the Swiss National Science Foundation (SNF, project number 200021 138068/1), and the Competence Centre for Materials Science and Technology (CCMX, project number 66) for financial support, and the European Synchrotron Radiation Facility (ESRF, Grenoble, France) for beam-time allocation at the beamlines ID24 and ID11. The support of Dr. S. A. J. Kimber, Mr. A. Marberger, Dr. M. Sridhar, Mr. P. Steiger during measurements is highly appreciated. V. M. thanks Dr. D. Mezza and Dr. D. Bartolini for fruitful discussions.

\section{Supporting Information Available}

The following files are available free of charge.

- SI.pdf: supporting information

This material is available free of charge via the Internet at http://pubs.acs.org/.

\section{References}

(1) Gleaves, J. T.; Ebner, J. R.; Kuechler, T. C. Catal. Rev. - Sci. Eng. 1988, 30, 49-116.

(2) Pérez-Ramírez, J.; Kondratenko, E. V. Catal. Today 2007, 121, 160-169. 
(3) Shannon, S. L.; Goodwin, J. G. Chem. Rev. 1995, 95, 677-695.

(4) Naphtali, L. M.; Polinski, L. M. J. Phys. Chem. 1963, 67, 369-375.

(5) Cavers, M.; Davidson, J. M.; Harkness, I. R.; Rees, L. C. V.; McDougall, G. S. J. Catal. 1999, 188, 426-430.

(6) Kritzenberger, J.; Wokaun, A. J. Molecular Catal. A 1997, 118, 235-245.

(7) Ortelli, E. E.; Wambach, J.; Wokaun, A. Appl. Catal. A 2000, 192, 137-152.

(8) Newton, M. A.; Belver-Coldeira, C.; Martínez-Arias, A.; Fernández-García, M. Nature Materials 2007, 6, 528-532.

(9) Newton, M. A.; Di Michiel, M.; Kubacka, A.; Iglesias-Juez, A.; Fernández-García, M. Angew. Chem. Int. Ed. 2012, 51, 2363-2367.

(10) Hartfelder, U.; Szlachetko, J.; Sa, J.; van Bokhoven, J. A. Analyst 2012, 137, 53745381.

(11) Valeur, B.; Berberan-Santos, M. N. Molecular Fluorescence: Principles and Applications, 2nd ed.; Wiley-VCH Verlag GmbH \& Co. KGaA: New York, United States of America, 2012.

(12) Baurecht, D.; Fringeli, U. P. Rev. Sci. Instrum. 2001, 72, 3782-3792.

(13) Stötzel, J.; Lützenkirchen-Hecht, D.; Frahm, R.; Grunwaldt, J. D. J. Phys.: Conf. Ser. 2013, 430, 012126.

(14) Szlachetko, J.; Ferri, D.; Marchionni, V.; Kambolis, A.; Safonova, O. V.; Milne, C. J.; Kröcher, O.; Nachtegaal, M.; Sa, J. J. Am. Chem. Soc. 2013, 135, 19071-19074.

(15) Bürgi, T.; Baiker, A. J. Phys. Chem. B 2002, 106, 10649-10658. 
(16) König, C. F. J.; van Bokhoven, J. A.; Schildhauer, T. J.; Nachtegaal, M. J. Phys. Chem. C 2012, 116, 19857-19866.

(17) Chiarello, G. L.; Ferri, D. Phys. Chem. Chem. Phys. 2015, 17, 10579-10591.

(18) Urakawa, A.; Bürgi, T.; Baiker, A. Chem. Phys. 2006, 324, 653-658.

(19) Urakawa, A.; Wirz, R.; Bürgi, T.; Baiker, A. J. Phys. Chem. B 2003, 107, 13061-13068.

(20) Wirz, R.; Bürgi, T.; Lindner, W.; Baiker, A. Anal. Chem. 2004, 76, 5319-5330.

(21) Kydd, R.; Ferri, D.; Hug, P.; Scott, J.; Teoh, W. Y.; Amal, R. J. Catal. 2011, 64, 277.

(22) Aguirre, A.; Collins, S. E. Catal. Today 2013, 205, 34-40.

(23) Marchionni, V.; Newton, M. A.; Kambolis, A.; Matam, S. K.; Weidenkaff, A.; Ferri, D. Catal. Today 2014, 229, 80-87.

(24) Ferri, D.; Matam, S. K.; Wirz, R.; Eyssler, A.; Korsak, O.; Hug, P.; Weidenkaff, A.; Newton, M. A. Phys. Chem. Chem. Phys. 2010, 12, 5634-5646.

(25) Ferri, D.; Newton, M. A.; Di Michiel, M.; Yoon, S.; Chiarello, G. L.; Marchionni, V.; Matam, S. K.; Aguirre, M. H.; Weidenkaff, A.; Wen, F.; Gieshoff, J. Phys. Chem. Chem. Phys. 2013, 15, 8629-8639.

(26) Nilsson, J.; Carlsson, P. A.; Fouladvand, S.; Martin, N. M.; Gustafson, J.; Newton, M. A.; Lundgren, E.; Grönbeck, H.; Skoglundh, M. ACS Catal. 2015, 5, 2481-2489.

(27) Urakawa, A.; Van Beek, W.; Monrabal-Capilla, M.; Galán-Mascarós, J. R.; Palin, L.; Milanesio, M. J. Phys. Chem. C 2011, 115, 1323-1329.

(28) Chernyshov, D.; van Beek, W.; Emerich, H.; Milanesio, M.; Urakawa, A.; Viterbo, D.; Palin, L.; Caliandro, R. Acta Crystallogr. A 2011, 67, 327-335. 
(29) Ferri, D.; Newton, M. A.; Di Michiel, M.; Chiarello, G. L.; Yoon, S.; Lu, Y.; Andrieux, J. Angew. Chem. Int. Ed. 2014, 53, 8890-8894.

(30) Allen, R. L.; Mills, D. W. Signal analysis: time, frequency, scale, and structure, 1st ed.; John Wiley \& Sons Inc.: New York, United States of America, 2004.

(31) Urakawa, A.; Bürgi, T.; Baiker, A. Chem. Eng. Sci. 2008, 63, 4902-4909.

(32) Kaszkur, Z. J. Appl. Crystall. 2000, 33, 1262-1270.

(33) König, C. F. J.; Schildhauer, T. J.; Nachtegaal, M. J. Catal. 2013, 305, 92-100.

(34) Lundgren, E.; Gustafson, J.; Mikkelsen, A.; Andersen, J. N.; Stierle, A.; Dosch, H.; Todorova, M.; Rogal, J.; Reuter, K.; Scheffler, M. Phys. Rev. Lett. 2004, 92, 046101.

(35) Westerstroem, R. et al. Phys. Rev. B 2011, 83, 115440.

(36) Datye, A. K.; Bravo, J.; Nelson, T. R.; Atanasova, P.; Lyubovsky, M.; Pfefferle, L. Appl. Catal. A 2000, 198, 179-196. 


\section{Graphical TOC Entry}

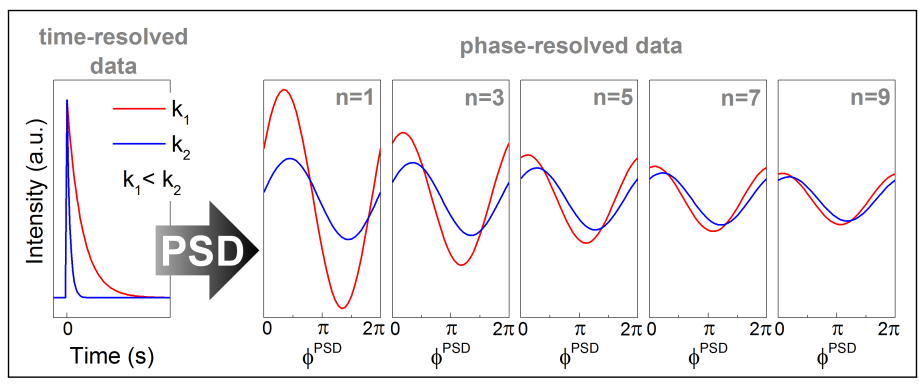

